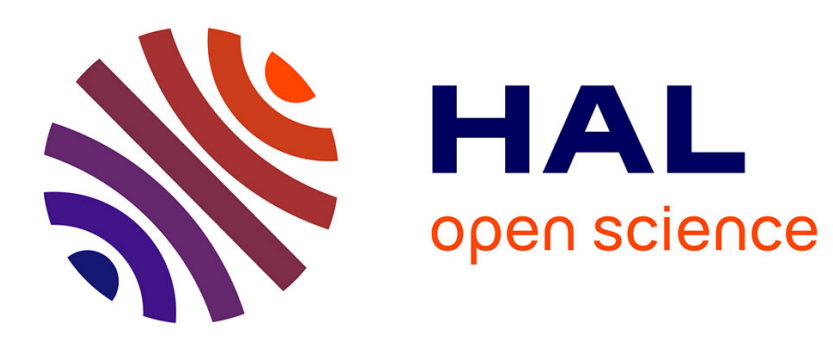

\title{
Subtleties in discrete-element modelling of wet granular soils
}

\author{
Jérôme Duriez, R. Wan
}

\section{To cite this version:}

Jérôme Duriez, R. Wan. Subtleties in discrete-element modelling of wet granular soils. Geotechnique, 2017, 67 (4), pp.365 - 370. 10.1680/jgeot.15.P.113 . hal-01868739

\section{HAL Id: hal-01868739 \\ https://hal.science/hal-01868739}

Submitted on 5 Sep 2018

HAL is a multi-disciplinary open access archive for the deposit and dissemination of scientific research documents, whether they are published or not. The documents may come from teaching and research institutions in France or abroad, or from public or private research centers.
L'archive ouverte pluridisciplinaire HAL, est destinée au dépôt et à la diffusion de documents scientifiques de niveau recherche, publiés ou non, émanant des établissements d'enseignement et de recherche français ou étrangers, des laboratoires publics ou privés. 


\title{
Subtleties in Discrete Element Modelling of wet granular soils
}

\author{
J. DURIEZ and R. WAN
}

The stress description provided by the discrete element method (DEM) when modelling wet granular materials is investigated for the case of low degrees of saturation within the pendular regime. The stress tensor as computed solely from contact and resultant capillary forces between individual pairs of particles is analysed in depth, being compared with an analytically derived stress expression that accounts for the distributed nature of internal actions such as the liquid pressure acting along wetted surfaces. Both fundamentally different approaches are shown to be equivalent, including for low suction values where wetted surfaces are significant and physical internal forces clearly deviate from the resultant point forces considered by DEM. Under wet conditions, it is demonstrated that the discrete element entity now includes both a particle and half a meniscus as a unit.

\section{INTRODUCTION}

A unified mechanical description of granular soils in biphasic (liquid saturated or dry) conditions and triphasic (unsaturated) conditions is still lacking, with the effective stress proposed by Bishop (1959) suffering shortcomings that have been amply discussed by Nuth \& Laloui (2008). In fact, the existence of an effective stress under unsaturated conditions had been challenged by Bishop himself (Bishop \& Blight, 1963). In this connection, refined micro-mechanical modelling investigations have been undertaken to gain insights in the mechanical behaviour of unsaturated granular soils (Chateau \& Dormieux, 2002; Hicher \& Chang, 2007; Wan et al., 2014), using in particular the Discrete Element Method (DEM, Cundall \& Strack, 1979) as a pertinent numerical tool for probing into the microstructure (Gili \& Alonso, 2002; Richefeu et al., 2006; Scholtès et al., 2009; Mani et al., 2013). A common feature of every DEM model is the consideration of particles interacting through solely point forces. Thus, DEM simulations of wet granular materials simulate directly the solid grains as discrete elements (DE's) and include the fluid phases by introducing extra point forces, compared with simulations in the dry state. These extra forces basically represent capillary forces resulting from the distribution of the liquid and gas phases. At low liquid content in the pendular regime, this spatial distribution takes the form of distinct liquid menisci between separated or contacting particles (Fig. 1).

Despite the description of internal forces with point-wise interaction forces, a total stress tensor can still be defined for DEM models and the objective of this Note is to discuss such a stress description from resultant capillary forces. In particular, we compare DEM with another approach based on analytical homogenization (Chateau et al., 2002). The analytical formulation is first recalled before elaborating the stress tensor expression for DEM models, which is thereafter compared with the homogenization approach, thus providing an extension of a preliminary comparison by Wan et al. (2015).

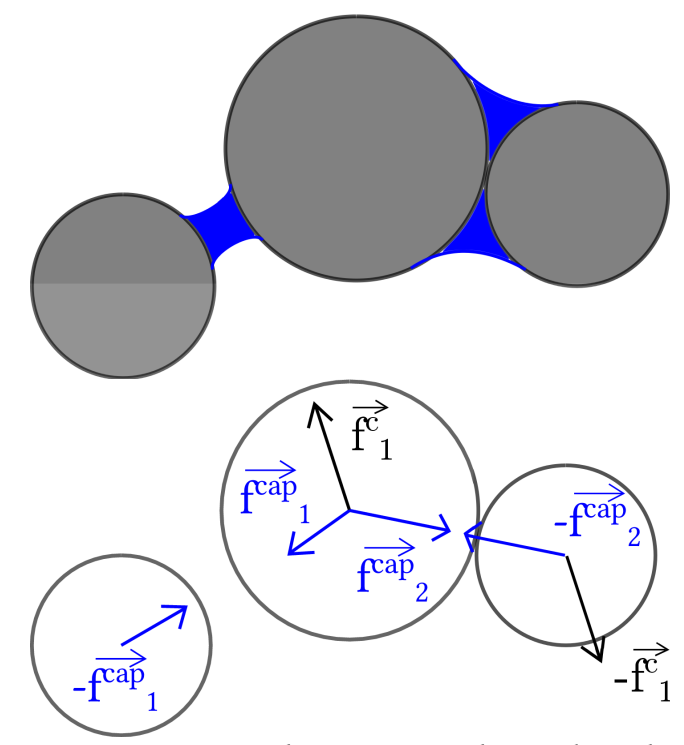

Fig. 1. Wet granular material with idealized spherical grains (top) and discrete elements sustaining both contact and capillary forces (bottom).

\section{ANALYTICAL HOMOGENIZATION OF WET GRANULAR SOILS}

The stress state of an unsaturated soil depends on the solid, liquid and gas phases involved, including the interfaces between them. In fact, liquid-gas interfaces and surface tension contribute necessarily to the total stress as they enter into the expression of the total energy of the unsaturated mixture (Nikooee et al., 2013; Madeo et al., 2013). Chateau \& Dormieux (2002), Chateau et al. (2002) derived the stress homogenization of a wet granular soil, expressing the total stress as a function of the 
various internal forces including an interfacial term:

$$
\boldsymbol{\Sigma}=\frac{1}{V}\left(\int_{V_{s}} \boldsymbol{\sigma} d V+\int_{V_{l}} \boldsymbol{\sigma} d V+\int_{V_{g}} \boldsymbol{\sigma} d V+\int_{S_{l g}} \pi d S\right)
$$

Eq. (1) accounts for surface tension internal forces along the interface $S_{l g}$ through the stress-like tensor $\pi$ that is expressed in terms of surface tension $\gamma$ and $\boldsymbol{\delta}_{\mathrm{lg}}$, the identity tensor restricted to $S_{l g}$ (Chateau et al., 2002). The magnitude of this stress contribution from interfaces has not yet been quantified, leading Wan et al. (2014) to neglect it in independent derivations. Also, Wan et al. (2015) did not include such surface term in a previous comparison with the stress expression for DEM models, that will be presented in the next section.

Accouting herein for all volume phases and this interface, we note that the stresses within the fluid phases are uniform pressure fields. As for the solid phase (the granular skeleton), the corresponding stress is defined from the stresses within grains (Bagi, 1996). This assumption may lead to stress components that are difficult to interpret when rotations of individual grains are taken into account (Nicot et al., 2013; Smith \& Wensrich, 2014); however such discussion is outside the scope of this Note. Eq. (1) is then rewritten, based on individual grain particles $p$ and converting the volume integrals into surface integrals, considering particle equilibrium without body forces (see e.g. Nicot et al., 2013):

$$
\begin{gathered}
\boldsymbol{\Sigma}=\frac{1}{V} \sum_{p} \int_{S_{p}}(\boldsymbol{\sigma} \vec{n}) \otimes \vec{x} d S+\frac{V_{l}}{V} u_{l} \boldsymbol{\delta} \\
+\frac{V_{g}}{V} u_{g} \boldsymbol{\delta}-\frac{1}{V} \int_{S_{l g}} \gamma \boldsymbol{\delta}_{l g} d S
\end{gathered}
$$

Eq. (2) involves tractions through $\boldsymbol{\sigma} \vec{n}$ and we note matric suction and surface tension respectively act along the wetted surface and the menisci contours where the three phases intersect, while contact forces are considered to act on vanishing surfaces (Fig. 2).

Finally, the total stress tensor is obtained as (see Chateau et al., 2002 and the Appendix):

$$
\begin{gathered}
\boldsymbol{\Sigma}=\frac{1}{V} \sum_{c 1,2} \vec{f}_{2}^{c} \otimes \vec{l}_{12}-s \chi-\boldsymbol{B}+u_{g} \boldsymbol{\delta} \\
\chi=\frac{1}{V}\left(V_{l} \boldsymbol{\delta}+\sum_{p} R_{p} \int_{S_{p}^{\prime}} \vec{n} \otimes \vec{n} d S\right) \\
\boldsymbol{B}=\frac{1}{V}\left(\sum_{p} R_{p} \int_{\Gamma_{p}} \vec{\gamma} \otimes \vec{n} d S+\int_{S_{l g}} \gamma \boldsymbol{\delta}_{l g} d S\right)
\end{gathered}
$$

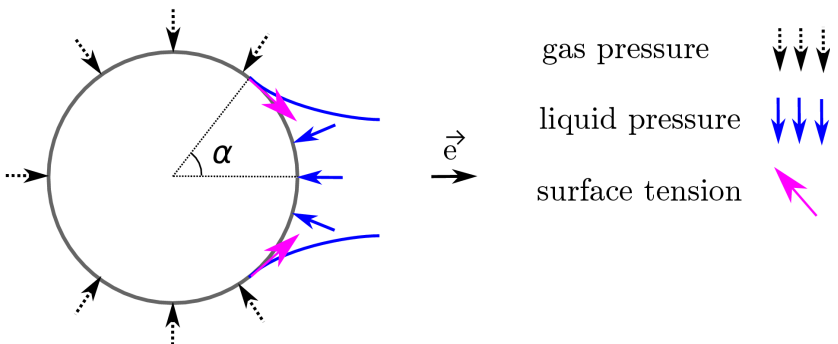

Fig. 2. Mechanical actions on the soil particles in wet conditions (point contact forces between particles are excluded).

\section{DEM STRESS MODELLING OF WET GRANULAR SOILS}

\section{DEM model formulation}

DEM models for wet granular soils usually differ through the expression of the capillary forces used to simulate the liquid menisci. For fully wettable particles where the contact angle is zero, the exact expression from matric suction and surface tension is:

$$
f^{\vec{c} a p}=\pi R \sin (\alpha)^{2}(2 \gamma+R s) \vec{e}
$$

Richefeu et al. (2006); Mani et al. (2013) used empirical relations to express directly the capillary force as functions of the particle distance and the meniscus volume. The present work adopts a model we developed within the Yade code (Šmilauer et al., 2010) from the one by Scholtès et al. (2009) that is based on systematic numerical solutions of Laplace (1806) equation as per Lian et al. (1993); Soulié et al. (2006).

Accordingly, the model includes capillary forces at each (separated or contacting) particle pair for which Laplace's equation can be solved, assuming a constant suction and a zero contact angle. The resulting liquid bridge configuration corresponds to uniform suction conditions, i.e. thermodynamic equilibrium.

In addition to these capillary forces, contact forces are applied at touching particles according to particle relative displacements and a standard elastic-plastic contact law. More details about the DEM model have been presented by Wan et al. (2015).

\section{Stress homogenization in DEM}

In addition to interaction forces, a stress tensor representation of the DEM model is still desirable for a meaningful analysis at the macroscopic level. Stress homogenization of the DEM assembly is again obtained from surface integral of external tractions acting on the discrete elements. Since the only 
external tractions are point forces in the DEM model, it follows immediately that:

$$
\Sigma=-\frac{1}{V} \sum_{p} \sum_{\vec{f}_{p}^{c}} \vec{f}_{p}^{c} \otimes \vec{x}
$$

It is herein noted that DEM simulations encompass only summations of forces and moments without the need for any information on the points of force application $\vec{x}$, if torques are disregarded.

For dry conditions, considering application points of contact forces to lie on the particle surfaces seems natural. In doing so, Eq. (5) leads to the homogenized contact stress:

$$
\Sigma=\boldsymbol{\sigma}^{\mathrm{cont}}=\frac{1}{V}\left(\sum_{c 1,2 \in V} \vec{f}_{2}^{c} \otimes \overrightarrow{l_{12}}-\sum_{p \in \partial V} \sum_{f^{\vec{e} \mathrm{t}}} \overrightarrow{f_{p}^{\mathrm{e} x t}} \otimes \vec{x}\right)
$$

The first term of Eq. (6) gathers contributions of every contact within the volume $V$. The second term involving external forces sustained by the particles at the boundaries is classically neglected (Nicot et al., 2013). For triaxial simulations with 20,000 particles, this induces a $2 \%$ discrepancy between the homogenized stresses and the ones deduced from the rigid boundaries used in the simulations (Fig. 3). Note that the perfect match between stresses deduced from Eq. (6) and at the boundaries demonstrates the quasi-static nature of the simulations.

In order to account for unsaturated conditions, Scholtès et al. (2009); Richefeu et al. (2006) supplemented the contact stress tensor with a so-called capillary stress tensor that includes the capillary forces such that $\boldsymbol{\Sigma}=\boldsymbol{\sigma}^{\text {cont }}+\boldsymbol{\sigma}^{\text {cap }}$. By analogy to the leading term of Eq. (6), the capillary stress tensor was assumed to take a similar form without any formal proof. Thus, summing over all menisci-bonded particle pairs:

$$
\boldsymbol{\sigma}^{\text {cap }}=\frac{1}{V} \sum_{m \in V} \vec{f}_{2}^{\overrightarrow{c a p}} \otimes \overrightarrow{l_{12}}
$$

The derivation of Eq. (7) from Eq. (5) would in fact require considering a common application point for $\overrightarrow{f_{1}^{c a p}}$ and $\overrightarrow{f_{2}^{c a p}}$ such that $\vec{x}_{1}-\vec{x}_{2}=\overrightarrow{l_{12}}$, but this might be considered as physically unrealistic for stretched menisci as seen in Fig. 4(a). A more physical choice as illustrated in Fig. 4(b) would lead to:

$$
\boldsymbol{\sigma}^{c a p}=-\frac{1}{V} \sum_{p \in V} \sum_{m \text { on } p} f_{p}^{\overrightarrow{c a p}} \otimes \vec{X}_{m}
$$
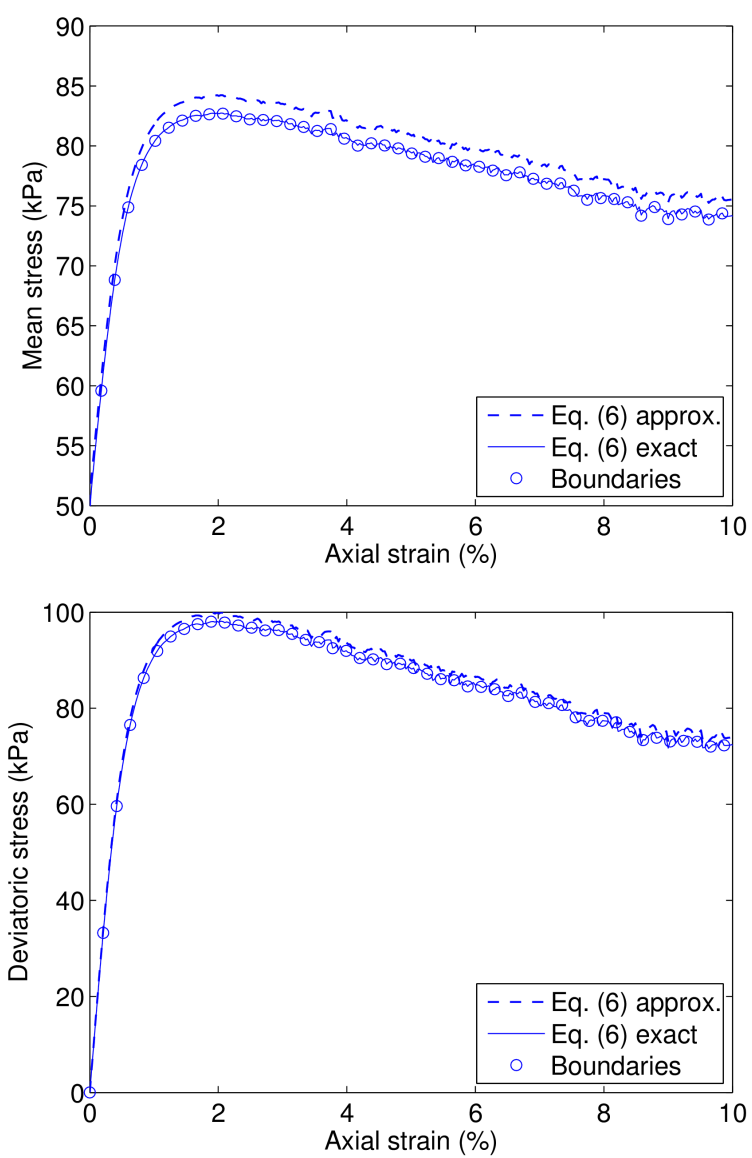

Fig. 3. Stress homogenization of the DEM model during triaxial loading (50 $\mathrm{kPa}$ confining pressure) in dry conditions. The second term of Eq. (6) is included for exact stress computations, and dropped for approx. ones.

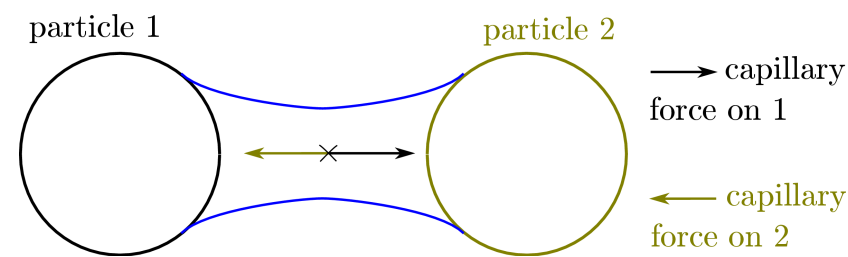

(a) Non-physical hypothesis underlying Eq. (7): particles sustain capillary forces that do not act on their surfaces

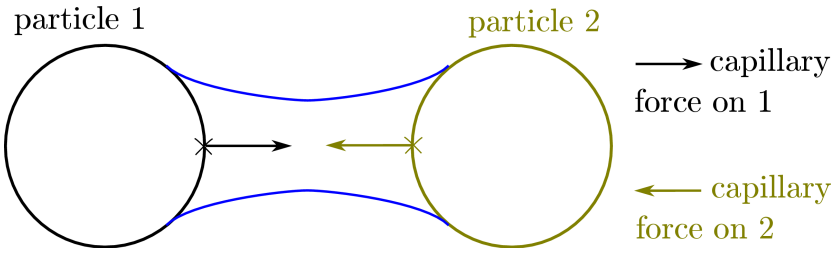

(b) Physical hypothesis underlying Eq. (8): particles sustain capillary forces that act on their surfaces

Fig. 4. Different possible application points of 
capillary forces for the stress homogenization of the DEM model.

As for the contact forces, the DEM model does not define any application points for capillary forces. It turns out that Eq. (7), Fig. 4(a), is finally more suitable to analyze the DEM model through an homogenized stress tensor. Indeed, $\boldsymbol{\sigma}^{c a p}$ as calculated from Eq. (7) is in closer agreement with $\left(\boldsymbol{\Sigma}-\boldsymbol{\sigma}^{\text {cont }}\right)$ than is $\boldsymbol{\sigma}^{c a p}$ as computed from Eq. (8); see Fig. 5. In this comparison, the total stress is deduced from the rigid boundaries, whereas the contact stress is calculated from Eq. (6).
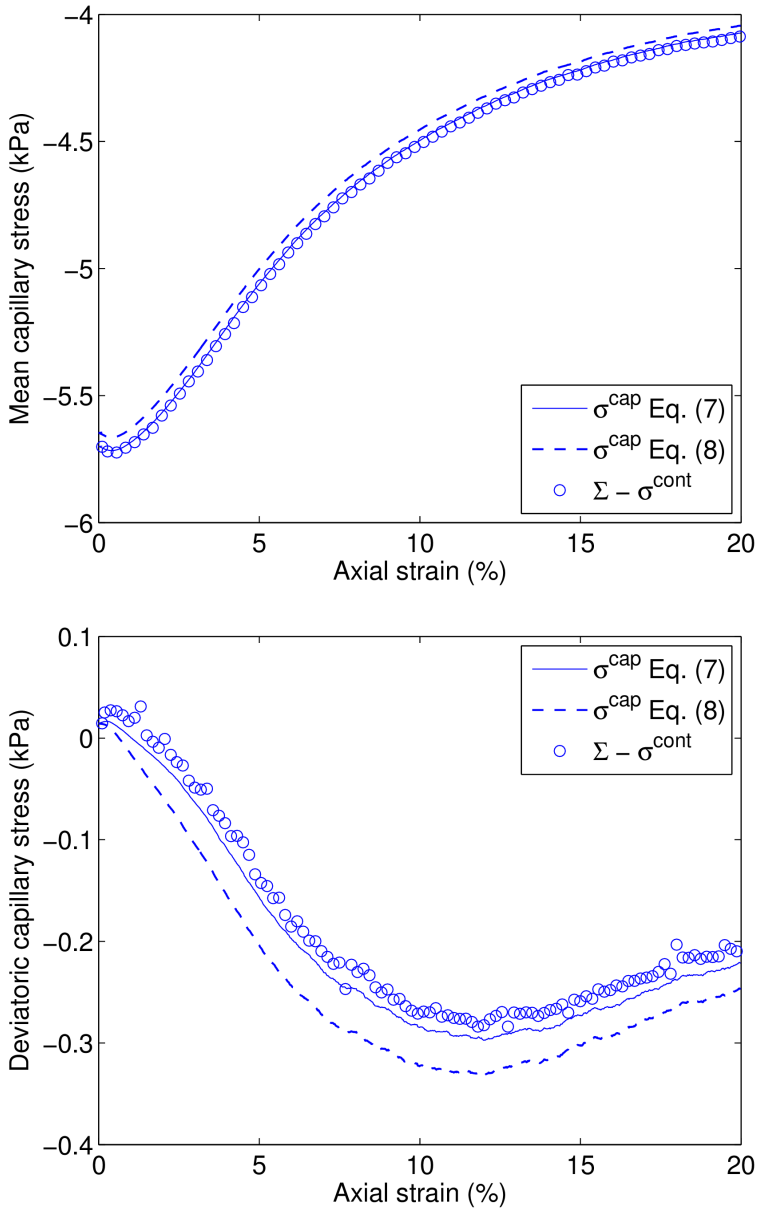

Fig. 5. Stress homogenization of the DEM model during triaxial loading (20 $\mathrm{kPa}$ confining pressure) in unsaturated conditions ( $5 \mathrm{kPa}$ suction ; $S_{r} \approx 21+/-5 \%$ ).

\section{STRESS DESCRIPTIONS: HOMOGENIZATION VS} DEM APPROACHES

\section{Preliminaries}

Following both the homogenization and DEM approaches, a capillary stress tensor appears as the difference between the total stress and the contact stress, neglecting the gas pressure. The capillary stresses as derived in the analytical approach is:

$$
\sigma_{\text {hom }}^{c a p}=\Sigma-\sigma^{\text {cont }}=-s \chi-B
$$

whereas, within the DEM framework,

$$
\boldsymbol{\sigma}_{D E M}^{c a p}=\Sigma-\sigma^{c o n t}=\frac{1}{V} \sum_{m \in V} \overrightarrow{f_{2}^{c a p}} \otimes \overrightarrow{l_{12}}
$$

A preliminary investigation on whether both modelling procedures provide equivalent stress desciptions of unsaturated granular materials was first undertaken by Wan et al. (2015), neglecting the interface stress contributions in $\sigma_{\text {hom }}^{\text {cap }}$. Numerical results along hydraulic loading paths showed discrepancies in the respective computed mean capillary stresses, which might be due to the following reasons:

(i) The DEM model does not account for the fluid volumes as such. Instead, it includes them indirectly through the resultant forces they induce on the solid phase.

(ii) Also, the DEM reduces the physical internal force distributions into resultant forces $\vec{f}=\int_{S} \boldsymbol{\sigma} \vec{n} d S$. Obviously, this has no impact on (rigid) particles displacements, and strains within granular soils. However, computing stresses involves the integral $\int_{S} \sigma \vec{n} \otimes \vec{x} d S$. Because the physical internal forces are reduced to a resultant point force, the stress computation in the DEM model leads to calculating $\vec{f} \otimes \vec{x}=\left(\int_{S} \sigma \vec{n} d S\right) \otimes \vec{x} \neq \int_{S} \sigma \vec{n} \otimes \vec{x} d S$.

The consistency between the two approaches is herein further investigated, starting from the exact expressions of the mean capillary stresses, i.e.

$$
\begin{aligned}
p_{\text {hom }}^{c a p} & =\frac{1}{3} \operatorname{tr}\left(\boldsymbol{\sigma}_{\text {hom }}^{\text {cap }}\right) \\
p_{D E M}^{c a p} & =\frac{1}{3} \operatorname{tr}\left(\boldsymbol{\sigma}_{D E M}^{c a p}\right)
\end{aligned}
$$

For sake of clarity, indices 2 and 12 are omitted in the DEM formulation, i.e.

$$
\begin{aligned}
p_{D E M}^{c a p} & =\frac{1}{3 V} \sum_{m} \operatorname{tr}\left(\overrightarrow{f^{c a p}} \otimes \vec{l}\right)=\frac{1}{3 V} \sum_{m} f^{\overrightarrow{c a p}} \cdot \vec{l} \\
& =-\frac{1}{3 V} \sum_{m} R_{1} \sin \left(\alpha_{1}\right)^{2}\left(2 \gamma+R_{1} s\right)\left(R_{1}+R_{2}-u_{n}\right) \\
& =-\frac{1}{3 V} \sum_{m} R_{2} \sin \left(\alpha_{2}\right)^{2}\left(2 \gamma+R_{2} s\right)\left(R_{1}+R_{2}-u_{n}\right)
\end{aligned}
$$

As for the analytical model, we get from Eq. (3) since $\vec{\gamma} \cdot \vec{n}=0$ for a zero contact angle: 


$$
\begin{aligned}
p_{\text {hom }}^{\text {cap }}= & -\frac{1}{3} \operatorname{tr}(s \boldsymbol{\chi})-\frac{1}{3} \operatorname{tr}\left(\frac{\gamma}{V} \int_{S_{l g}} \boldsymbol{\delta}_{l g} d S\right) \\
= & -\frac{s}{3 V}\left(V_{l} \operatorname{tr}(\boldsymbol{\delta})+\sum_{p} R_{p} \int_{S_{p}^{l}} \operatorname{tr}(\vec{n} \otimes \vec{n}) d S\right) \\
& -\frac{\gamma}{3 V} S_{l g} \operatorname{tr}\left(\boldsymbol{\delta}_{l g}\right) \\
= & -\frac{1}{3 V}\left[s\left(3 V_{l}+\sum_{p} R_{p} S_{p}^{l}\right)+2 \gamma S_{l g}\right] \\
= & -\frac{1}{3 V} \sum_{m}\left[s\left(3 V_{m}+\sum_{p=1}^{2} R_{p}{ }^{3}\left(1-\cos \alpha_{p}\right)\right)+2 \gamma S_{m}\right]
\end{aligned}
$$

It is easy to show that the two approaches secure agreement in the case of high suction, i.e. a low degree of saturation, as observed by Wan et al. (2015). In such a case, filling angles are small, suction terms outweight surface tension terms in Eq. (13) and menisci volume and surface are negligible in Eq. (14). Finally, interparticle penetration can be considered as small, which is classical in DEM for contacting quasi-rigid spheres, and also occurs for noncontacting ones as menisci break after a small gap under high suction. Thus, a common expression is finally obtained:

$$
p_{D E M}^{\text {cap }} \approx p_{\text {hom }}^{\text {cap }} \approx \frac{-2}{3 V} s \sum_{m} \pi R_{m}{ }^{3} \alpha_{m}{ }^{2}
$$

Indeed, the two discrepancies reasons alluded to previously disappear for very low saturation. In particular, negligible wetted surfaces $S$ lead to $\left(\int_{S} \sigma \vec{n} d S\right) \otimes \vec{x} \approx \int_{S} \sigma \vec{n} \otimes \vec{x} d S$.

Due to intricacies through Laplace's equation between the menisci geometrical variables and the surface tension and suction, an equivalence between Eq. (13) and (14) cannot be ruled out for a higher saturation where physical internal forces are distributed along significant surfaces. Such case is thus numerically investiguated in the following.

\section{Examples}

The capillary stress tensors expressed in Eqs. (9) and (10) are now compared numerically considering the DEM simulation of axisymmetric stress loading at constant suction, imposed on a numerical specimen of dense silt-like packing with $58 \mu \mathrm{m}$ as mean diameter, see Wan et al. (2015) for details. While obtaining $\sigma_{D E M}^{c a p}$ from the DEM model is straightforward, the computed fluid phase distribution at the same time enables the determination of $\boldsymbol{\sigma}_{\mathrm{hom}}^{\text {cap }}$. Interpreting the DEM model through the analytical approach i.e. $\boldsymbol{\sigma}_{\text {hom }}^{\text {cap }}$ aims at verifying whether the stress state of the DEM model i.e. $\sigma_{D E M}^{c a p}$ coincides with the stress state of the simulated wet granular system.

In spite of the fundamental differences in paradigm between the two approaches, Fig. 6 shows that the capillary stresses as computed from DEM and homogenization approaches do coincide for both low and high suctions values. The suction values here considered, 10 and $300 \mathrm{kPa}$, respectively correspond to saturation between $8 \%$ and $12 \%$, or $0.03 \%$ and $0.055 \%$, and to filling angle distributions around $30^{\circ}$ or $8^{\circ}$.
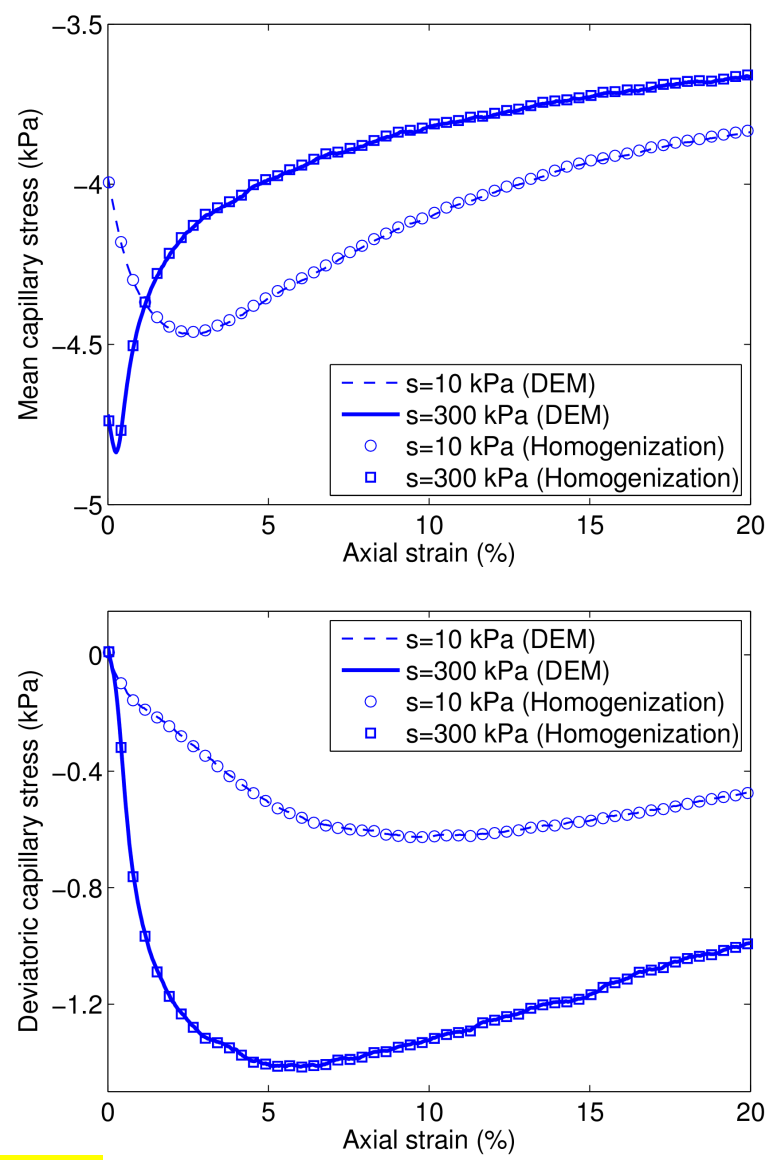

Fig. 6. Capillary stresses for triaxial loading with 10 $\mathrm{kPa}$ of confining pressure and different suctions. Capillary stresses are computed from the surface and lineal distributions through analytical homogenization, Eq. (9), or from resultant forces, Eq. (10), through DEM.

Such equivalence between $\sigma_{\text {hom }}^{\text {cap }}$ and $\sigma_{D E M}^{c a p}$ tensors for any suction implies that the DE's do not correspond only to solid particles, but also encompass halves of the menisci volume and surface, so that a complete stress description can be 
obtained from the DE's only. This surprising property of DE's in models of wet granular materials is related to the relevancy of considering a common application point for the capillary forces, as shown in the above.

Note that obtaining such above equivalence, which was previously suggested by Chateau et al. (2002) without much formal justication, is not possible if interfaces are neglected (Wan et al., 2015).

\section{CONCLUSIONS}

Wet granular materials include internal forces that are distributed in nature, such as liquid pressures or surface tension acting along wetted surfaces or interfaces. However, the Discrete Element Method still shows a consistent stress description from resultant point forces for any saturation in the pendular regime. Indeed, the DE's of corresponding DEM models encompass fluid volumes and interface surfaces in addition to the solid particles volumes.

The present work is currently being extended to consider non-zero contact angles and surface tension along every interface.

\section{APPENDIX: TOTAL STRESS DERIVATION FOR A} WET GRANULAR SOIL

The explicit derivation of Eq. (3) from (2) is obtained by taking into account all external tractions acting on particles, with $\sigma \vec{n}=-\vec{t}$ due to the considerations of external normals in the divergence theorem. Furthermore, all vectors $\vec{x} \in S_{p}$ are expressed from the particles centers: $\vec{x}=R_{p} \vec{n}$; this choice being immaterial by virtue of the particles' equilibrium. Then,

$$
\begin{aligned}
& \boldsymbol{\Sigma}+\frac{1}{V} \int_{S_{l g}} \gamma \boldsymbol{\delta}_{l g} d S=\frac{1}{V}\left(\sum_{p} \int_{S_{p}}-\vec{t} \otimes \vec{n} R_{p} d S+V_{l} u_{l} \boldsymbol{\delta}\right. \\
& +V_{g} u_{g} \delta \\
& =\frac{1}{V} \sum_{p}\left(\int_{S_{p}^{\prime}} u_{l} \vec{n} \otimes \vec{n} R_{p} d S+\int_{S_{p} \backslash S_{p}^{\prime}} u_{g} \vec{n} \otimes \vec{n} R_{p} d S\right. \\
& \left.-\int_{\Gamma_{p}} \vec{\gamma} \otimes \vec{n} R_{p} d l-\sum_{c} \vec{f}_{p}^{c} \otimes \vec{n} R_{p}\right) \\
& +\frac{V_{l}}{V} u_{l} \boldsymbol{\delta}+\frac{V_{g}}{V} u_{g} \delta \\
& =\frac{1}{V} \sum_{p}\left(R_{p}\left(u_{l}-u_{g}\right) \int_{S_{p}^{\prime}} \vec{n} \otimes \vec{n} d S+u_{g} R_{p} S_{p} \frac{\delta}{3}\right. \\
& \left.-R_{p} \int_{\Gamma_{p}} \vec{\gamma} \otimes \vec{n} d l-\sum_{c} R_{p} \vec{f}_{p}^{c} \otimes \vec{n}\right) \\
& +\frac{V_{l}}{V}\left(u_{l}-u_{g}\right) \delta+\frac{V_{g}+V_{l}}{V} u_{g} \delta
\end{aligned}
$$

$$
\begin{aligned}
= & \frac{1}{V} \sum_{p}\left(-R_{p} s \int_{S_{p}^{\prime}} \vec{n} \otimes \vec{n} d S+u_{g} V_{p} \boldsymbol{\delta}\right. \\
& \left.\quad-R_{p} \int_{\Gamma_{p}} \vec{\gamma} \otimes \vec{n} d l-\sum_{c} R_{p} \vec{f}_{p}^{c} \otimes \vec{n}\right) \\
& -\frac{V_{l}}{V} s \boldsymbol{\delta}+\frac{V-V_{s}}{V} u_{g} \delta \\
= & -s \frac{1}{V}\left(V_{l} \delta+\sum_{p} R_{p} \int_{S_{p}^{\prime}} \vec{n} \otimes \vec{n} d S\right)+u_{g} \delta \\
& -\frac{1}{V} \sum_{p} R_{p} \int_{\Gamma_{p}} \vec{\gamma} \otimes \vec{n} d l-\frac{1}{V} \sum_{p} \sum_{c} R_{p} \vec{f}_{p}^{c} \otimes \vec{n}
\end{aligned}
$$

The last contact force term is classically rewritten summing over all binary contacts $(1,2)$, which

\begin{tabular}{|c|c|}
\hline $\boldsymbol{B}$ & $\begin{array}{l}\text { capillary stress contribution } \\
\text { proportional to } \gamma\end{array}$ \\
\hline$\vec{e}$ & meniscus orientation \\
\hline $\overrightarrow{f_{p}^{c}}, \overrightarrow{f_{p}^{c a p}}$ & $\begin{array}{l}\text { contact or capillary force } \\
\text { sustained by particle } p\end{array}$ \\
\hline$\vec{n}$ & solid outward normal \\
\hline$p_{D E M}^{c a p}, p_{\text {hom }}^{c a p}$ & mean pressure of $\sigma_{D E M}^{c a p}, \sigma_{h o m}^{c a p}$ \\
\hline$R_{1}, R_{2}, R_{p}$ & radii of particles $1,2, p$ \\
\hline$R_{m}$ & $\begin{array}{l}\text { mean radius of a liquid bridge } \\
\text { bonded pair }\end{array}$ \\
\hline$S_{l g}, S_{m}$ & $\begin{array}{l}\text { liquid-gas interface surface at the } \\
\text { sample or menisci scale }\end{array}$ \\
\hline$S_{p}, S_{p}^{l}$ & $\begin{array}{l}\text { total or wetted surface of solid } \\
\text { particle } p\end{array}$ \\
\hline$S_{r}$ & saturation ratio \\
\hline$s=u_{g}-u_{l}$ & matric suction \\
\hline$\vec{t}$ & tractions on solid particles \\
\hline$u_{n}$ & $\begin{array}{l}\text { overlap between discrete } \\
\text { elements: positive for geometrical }\end{array}$ \\
\hline
\end{tabular}
demonstrates Eq. (3).

\section{ACKNOWLEDGMENTS}

This work is supported by the Natural Science and Engineering Research Council of Canada and Foundation Computer Modelling Group within the framework of a Government-Industry Partnership (NSERC-CRD) Grant. The authors also acknowledge gratefully various discussions of ideas with Félix Darve and Vincent Richefeu from Laboratoire 3SR and François Nicot from IRSTEA, all in Grenoble, France.

\section{LIST OF NOTATIONS}


Methods in Geomechanics 26, No. 8, 831-844, doi:10.1002/nag.227.

Chateau, X., Moucheront, P. \& Pitois, O. (2002). Micromechanics of Unsaturated Granular Media. Journal of Engineering Mechanics 128, No. 8, 856863, doi:10.1061/(ASCE)0733-9399(2002)128:8(856)

Cundall, P. \& Strack, O. (1979). A discrete numerical model for granular assemblies. Géotechnique 29, 4765.

Gili, J. A. \& Alonso, E. E. (2002). Microstructural deformation mechanisms of unsaturated granular soils. International fournal for Numerical and Analytical Methods in Geomechanics 26, No. 5, 433468, doi:10.1002/nag.206.

Hicher, P.-Y. \& Chang, C. (2007). A microstructural elastoplastic model for unsaturated granular materials. International fournal of Solids and Structures 44, No. 78, 2304 - 2323, doi:10.1016/j.ijsolstr.2006.07.007.

Laplace, P. S. (1806). Sur l'action capillaire in Supplément au livre $X$ du traité de mécanique céleste. Duprat (Paris), available in Oeuvres complètes de Laplace, Tome 4, on www.gallica.bnf.fr.

Lian, G., Thornton, C. \& Adams, M. J. (1993). A theoretical study of the liquid bridge forces between two rigid spherical bodies. Fournal of Colloid and Interface Science 161, No. 1, 138 - 147, doi:10.1006/jcis.1993.1452.

Madeo, A., dell'Isola, F. \& Darve, F. (2013). A continuum model for deformable, second gradient porous media partially saturated with compressible fluids. Journal of the Mechanics and Physics of Solids 61, No. 11, 2196 - 2211, doi:10.1016/j.jmps.2013.06.009.

Mani, R., Kadau, D. \& Herrmann, H. J. (2013). Liquid migration in sheared unsaturated granular media. Granular Matter 15, No. 4, 447-454, doi:10.1007/s10035-012-0387-3.

Nicot, F., Hadda, N., Guessasma, M., Fortin, J. \& Millet, O. (2013). On the definition of the stress tensor in granular media. International fournal of Solids and Structures 50, No. 1415, 2508 - 2517, doi:10.1016/j.ijsolstr.2013.04.001.

Nikooee, E., Habibagahi, G., Hassanizadeh, S. \& Ghahramani, A. (2013). Effective stress in unsaturated soils: A thermodynamic approach based on the interfacial energy and hydromechanical coupling. Transport in Porous Media 96, No. 2, 369396, doi:10.1007/s11242-012-0093-y.

Nuth, M. \& Laloui, L. (2008). Effective stress concept in unsaturated soils: Clarification and validation of a unified framework. International fournal for Numerical and Analytical Methods in Geomechanics 32, No. 7, 771-801, doi:10.1002/nag.645. 
Richefeu, V., El Youssoufi, M. S. \& Radjaï, F. (2006). Shear strength properties of wet granular materials. Phys. Rev. E 73, 051304-1-051304-11, doi:10.1103/PhysRevE.73.051304.

Scholtès, L., Hicher, P.-Y., Nicot, F., Chareyre, B. \& Darve, F. (2009). On the capillary stress tensor in wet granular materials. International fournal for Numerical and Analytical Methods in Geomechanics 33, No. 10, 1289-1313, doi:10.1002/nag.767.

Smith, A. \& Wensrich, C. (2014). The effects of particle dynamics on the calculation of bulk stress in granular media. International fournal of Solids and Structures 51, No. 2526, 4414 - 4418, doi:10.1016/j.ijsolstr.2014.09.008.

Soulié, F., Cherblanc, F., El Youssoufi, M. \& Saix, C. (2006). Influence of liquid bridges on the mechanical behaviour of polydisperse granular materials. International fournal for Numerical and Analytical Methods in Geomechanics 30, No. 3, 213-228, doi:10.1002/nag.476.

Šmilauer, V., Catalano, E., Chareyre, B., Dorofeenko, S., Duriez, J., Gladky, A., Kozicki, J., Modenese, C., Scholtès, L., Sibille, L., Stránský, J. \& Thoeni, K. (2010). Yade Documentation. 1st edn., The Yade Project, http://yade-dem.org.

Wan, R., Duriez, J. \& Darve, F. (2015). A tensorial description of stresses in triphasic granular materials with interfaces. Geomechanics for Energy and the Environment 4, 73 - 87, doi:10.1016/j.gete.2015.11.004.

Wan, R., Khosravani, S. \& Pouragha, M. (2014). Micromechanical analysis of force transport in wet granular soils. Vadose Zone fournal 13, No. 5, 1-12, doi:10.2136/vzj2013.06.0113. 